\title{
Combination of Rehabilitative Therapy with Ultramicronized Palmitoylethanolamide for Chronic Low Back Pain: An Observational Study
}

\author{
Dalila Scaturro - Chiara Asaro - Lorenza Lauricella - Sofia Tomasello • \\ Giustino Varrassi · Giulia Letizia Mauro
}

Received: October 29, 2019 / Published online: December 20, 2019

(C) The Author(s) 2019

\begin{abstract}
Introduction: Chronic low back pain (LBP) caused by intervertebral disc herniation was reported in the 2010 Global Burden of Disease study to be the main reason for years lived with disability. It causes significant personal, social, and economic burdens. Many of those who suffer from LBP find conventional medical treatments to be unsatisfactory for treating their pain, so they are increasingly resorting to complementary and alternative medicine (CAM) therapies. Given that the population is aging, there is an urgent need to characterize the combinations of complementary therapies that yield the best outcomes and treatments, even for prolonged periods. This observational
\end{abstract}

Enhanced Digital Features To view enhanced digital features for this article go to https://doi.org/10.6084/ m9.figshare.11295302.

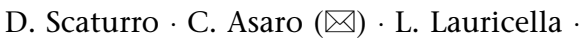

G. Letizia Mauro

Department of Physical Medicine and

Rehabilitation, University Hospital "P. Giaccone",

Palermo, Italy

e-mail: chiarasaro@virgilio.it

S. Tomasello

School of Medicine and Surgery, University of

Palermo, Palermo, Italy

G. Varrassi

Paolo Procacci Foundation, Via Tacito 7,

00193 Rome, Italy study aimed to evaluate the effect of ultramicronized palmitoylethanolamide (umPEA) + CAM (daily functional rehabilitation + decontracting massage) therapies on chronic pain in patients suffering from multiple herniated discs in the lumbar spine.

Methods: Eligible patients received $600 \mathrm{mg}$ of umPEA twice a day in combination with a daily functional rehabilitation session according to the McKenzie Method of Mechanical Diagnosis and Therapy plus a decontracting massage for 20 consecutive days, followed by $600 \mathrm{mg}$ of umPEA once a day for 40 days in addition to standard therapy.

Results: The results showed that the average pain intensity score, evaluated via the Numeric Rating Scale, progressively decreased during the study period, reaching a value that was not clinically relevant at the end of the observation period. Pain relief was paralleled by improvements in the physical and mental components of quality of life as evaluated with the SF-36 questionnaire as well as in disability for low back pain as evaluated with the Oswestry Disability Questionnaire. Collectively, the results demonstrate that umPEA in combination with CAM therapies could be an important strategy for combating LBP.

Conclusions: The multiple action of PEA in combination with CAM therapies may represent the multitarget approach needed to tackle the as-yet unsolved problem of chronic pain resistant to conventional therapies. 
Keywords: Chronic low back pain; Complementary and alternative medicine; Multidisciplinary therapies; Multitarget approach; Ultramicronized palmitoylethanolamide

\section{Key Summary Points}

Chronic low back pain (LBP) causes significant personal, social, and economic burdens.

Conventional medical treatments for LBP are frequently unsatisfactory, so patients resort to complementary and alternative medicine (CAM) therapies.

Treatment with ultramicronized palmitoylethanolamide in combination with daily functional rehabilitation and decontracting massage in patients affected by multiple herniated discs in the lumbar spine elicited progressive pain relief paralleled by improvements in the physical and mental components of quality of life.

The multiple action of palmitoylethanolamide in combination with CAM therapies may represent the multitarget approach needed to tackle the as-yet unsolved problem of chronic pain resistant to conventional therapies.

\section{INTRODUCTION}

Intervertebral disc herniation can cause back pain and/or radiculopathy, and it accounts for a significant proportion of the patients who have spinal surgery around the world. Lumbar disc herniation (LDH) is the most common cause of low back pain (LBP) and sciatica. It occurs in around $9 \%$ of all people worldwide, impacts substantially on their quality of life, and represents a significant economic burden $[1,2]$. In recent years, lifestyle changes have led to a gradual increase in the incidence of LDH, and the mean age of onset has become younger. In industrialized countries, LBP and sciatica are among the leading causes of work incapacity and disability before the age of 45 [3]. LDH has been associated with disruption of the annulus fibrosus, extrusion of the nucleus pulposus, and stimulation of nerve fibers, leading to pain [2]. LBP consequent to LDH was reported to be the main cause of years lived with disability in the 2010 Global Burden of Disease study, confirming that it is a disease that causes significant personal, social, and economic burdens [4]. People with back pain suffer from worse physical and mental health than people without back pain.

Recommended treatments for LDH include surgery and conservative methods such as transforaminal epidural steroid injection [5]. However, injections have been linked with various serious adverse events. In addition, although early surgical intervention is effective at alleviating sciatica earlier than conservative treatment, the results of surgical intervention and conservative treatment were found to be similar from one year onwards [6], and cases of failed back surgery syndrome where chronic pain persists after surgery continue to be reported [7]. For these reasons, complementary and alternative medicine (CAM) therapies are gaining ever-greater recognition as a means to treat back pain. According to the 2002 National Health Interview Survey of the resident civilian noninstitutionalized US population, the majority of the respondents who used complementary and alternative medicine therapies for back pain perceived a benefit [8]. Indeed, the updated 2017 LBP guidelines of the American College of Physicians recommend the use of nonpharmacologic treatments for patients with LBP. Guidelines also recommend the use of exercise in combination with other nonpharmacological therapies such as massage and nutraceuticals with known pain-relieving activity. One biomolecule that has been used in the management of chronic pain is palmitoylethanolamide (PEA). PEA is an acylethanolamide that is widely distributed in different tissues, including nervous tissues, and is synthesized on demand [9]. Evidence indicates that PEA is an important anti-inflammatory, 
analgesic, and neuroprotective mediator that acts at several molecular targets in both the central and peripheral nervous systems [10]. In addition to considerable evidence from experimental studies, several clinical studies published in in recent years have confirmed that PEA has anti-inflammatory and pain-relieving properties in humans [10, 11]. Most clinical trials with PEA have focused on formulations with particles that were subjected to the socalled fluid jet micronization process. This yields particles with a defined size profile (6-10 $\mu \mathrm{m}$ at most) that is completely different from and statistically lower than that of naïve PEA (in the 100-700 $\mu \mathrm{m}$ range). This smaller size (implying a higher surface-to-volume ratio) along with increased potential energy of the particles leads to better solute solubility. These characteristics result in better diffusion and distribution of micronized and ultramicronized PEA compared to the naïve form, and thus superior biological efficacy $[10,12]$. The properties of umPEA prompted us to investigate the effect of umPEA + CAM (daily functional rehabilitation and decontracting massage) therapies on chronic pain in patients suffering from multiple herniated discs in the lumbar spine.

\section{METHODS}

A prospective observational study was carried out between March 2016 and April 2017 of outpatients at the Complex Operating UnitRehabilitation, Polyclinic University Hospital Paolo Giaccone, Palermo (Italy). Patients aged $\geq 18$ years old who were suffering from chronic pain due to multiple herniated discs in the lumbar spine were enrolled. Patient diagnoses were checked by RMN. At the time of enrollment (baseline), the patients were being treated with analgesics, albeit with poor results. However, they maintained this therapeutic pattern, based generally on gabapentinoids or opioids, during the study.

Standard treatment was supplemented with $600 \mathrm{mg}$ of umPEA (Normast ${ }^{\circledR} 600 \mathrm{mg}$, Epitech Group SpA, Saccolongo, Italy) twice a day in combination with a daily functional rehabilitation session according to the McKenzie Method of Mechanical Diagnosis and Therapy (MDT) $[13,14]$ plus a decontracting massage for 20 consecutive days, and subsequently $600 \mathrm{mg}$ of umPEA once a day for 40 days. Pain intensity was evaluated via the Numeric Rating Scale (NRS), which consists of a 11-point grading scale (0-10) in which a score of 0 represents the total absence of pain and 10 corresponds to the presence of the worst pain imaginable. Quality of life was evaluated via the 36-Item Short Form Health Survey questionnaire (SF-36) [15]. Disability elicited by low back pain was evaluated by the Oswestry Disability Questionnaire (ODQ) [16].

Statistical analysis was performed using the generalized linear mixed model (GLMM). Variables such as gender and age were included in the model as covariates. Data are given as the mean \pm standard error (SE) unless otherwise stated. A $p$ value of less than 0.05 was considered significant.

This study was performed in accordance with the Good Clinical Practice guidelines and the principles of the Declaration of Helsinki of 1964 and its subsequent revisions. The study was approved by the Polyclinic University Hospital Paolo Giaccone Ethics Committee (report number 05/2019). Written consent for participation was obtained from each patient after they had been fully informed of the purpose of the study.

\section{RESULTS}

One hundred twenty patients of both genders (37 males and 83 females) with a mean age of $56.4 \pm 13.0$ who were suffering from lumbosciatica (95) and lumbocruralgia due to multiple herniated discs in the lumbar spine (25) were eligible for statistical analysis. The patients reported painful symptoms suggestive of neuropathic, neurogenic, or radicular pain. They had experienced pain for about 2 years, even though they were being treated with pregabalin ( $n=87 ; 75 \mathrm{mg}, 1$ capsule 2 times a day) or oxycodone $(n=33 ; 5 \mathrm{mg}, 1$ capsule 2 times a day) as their standard therapy.

With the addition of UmPEA + CAM therapies to their standard therapy, their average 
pain intensity scores decreased significantly from $6.3 \pm 0.1$ at baseline to $3.7 \pm 0.09$ and $2 \pm 0.09$ at 30 and 60 days, respectively $(p<0.001$; Fig. 1$)$.

The impact of chronic pain on quality of life changed significantly during the course of umPEA + CAM therapies. The physical component (PCS-36) increased from $30.7 \pm 0.3$ at baseline to $37.1 \pm 0.3$ and $43.9 \pm 0.4$ at 30 and 60 days, respectively. The increase in PCS-36 score was already statistically significant after 30 days of treatment $(p<0.0001$ for 30-day vs baseline scores), and a further increase occurred after 60 days of treatment $(p<0.0001$ for 30 -day vs 60 -day scores). $p$ values were evaluated at net of age, which was found to influence the response $(p=0.0009)$; see Table 1 .

The mental component (MCS-36) was also improved by the treatment; the MCS-36 score was $31.4 \pm 0.2$ at baseline and increased to $38.1 \pm 0.4$ and $44.1 \pm 0.4$ at 30 and 60 days, respectively (Table 1 ). Similarly to the PCS-36 score profile, the increase in score was already statistically significant after 30 days of treatment ( $p<0.0001$ for 30-day vs baseline scores), and a further increase occurred after 60 days of treatment $(p<0.0001$ for 30-day vs 60-day

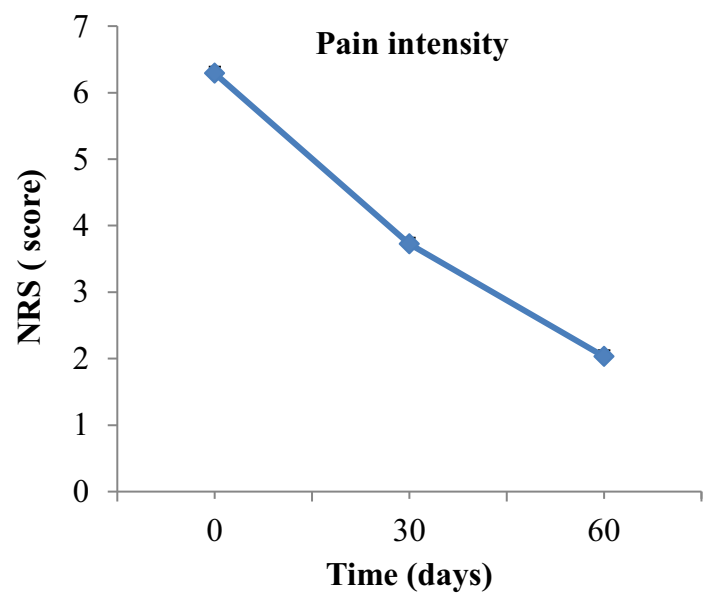

Fig. 1 Effect of adjuvant umPEA + CAM therapies on pain intensity scores in patients with chronic pain caused by lumbosciatica or lumbocruralgia due to multiple herniated discs in the lumbar spine. Data are expressed as the mean \pm SE; $n=120$. Note that the pain intensity score decreased with treatment over time: $p<0.0001$ scores). The influence of the covariate age was not relevant to the response $(p=0.06)$.

Disability for low back pain in patients undergoing umPEA + CAM therapies significantly decreased from $44.2 \pm 0.4$ at baseline to $37.4 \pm 0.3$ and $28.7 \pm 0.4$ at 30 and 60 days, respectively $(p<0.001$, Table 1$)$. Statistical analysis showed that disability improved significantly over time $(p<0.0001)$ and that the effect was already significant after 30 days of treatment $(p<0.0001$ for 30-day vs baseline scores), with a further increase after 60 days of treatment $(p<0.0001$ for 30-day vs 60-day scores; Table 1). $p$ values were evaluated at net of age, which was found to influence the response $(p=0.0002)$.

\section{DISCUSSION}

The results obtained in this observational study confirmed that umPEA + CAM therapies might be beneficial to patients with LBP, given that the average pain intensity score decreased progressively during the study period and reached a value that was not clinically relevant at the end of the observation period. Improvements in the physical and mental components of quality of life as well as disability for low back pain provide further confirmation of the beneficial effect of umPEA + CAM therapies.

LBP is a major public health problem, and has been identified as a leading cause of disability worldwide. Persistent LBP is associated with significant individual functional impairment, high utilization of health care, work absenteeism, and long-term incapacity [4]. Many people who suffer from LBP have found conventional medical treatments to be ineffective and unreliable for treating their pain; examples include the patients enrolled in the present study, who were being treated with conventional therapies that had not significantly resolved their chronic pain. Because of this, a large proportion of the population with LBP have used some form of CAM therapy in the past year, with particularly high use reported among those with limiting back pain, who perceive a great deal of benefit from CAM [17]. Current guidelines for chronic LBP recommend 
Table 1 Effect of adjuvant umPEA + CAM therapies on quality of life and disability for low back pain in patients with chronic pain caused by lumbosciatica or lumbocruralgia due to multiple herniated discs in the lumbar spine

\begin{tabular}{lllll}
\hline & \multicolumn{2}{l}{ Time (days) } & \multicolumn{1}{c}{$\boldsymbol{p}^{*}$} \\
\cline { 2 - 4 } & $\mathbf{0}$ & $\mathbf{3 0}$ & $\mathbf{6 0}$ & $<0.0001$ \\
\hline PCS-F36 & $30.7 \pm 0.3$ & $37.1 \pm 0.3$ & $43.9 \pm 0.4$ & $<0.0001$ \\
MCS-F36 & $31.4 \pm 0.2$ & $38.1 \pm 0.4$ & $44.1 \pm 0.4$ & $<0.0001$ \\
ODQ & $44.2 \pm 0.4$ & $37.4 \pm 0.3$ & $28.7 \pm 0.3$ & \\
\hline
\end{tabular}

Data (scores) are expressed as the mean \pm SE; $n=120$ at each time point

PCS-F36 physical health, MCS-F36 mental health, ODQ Oswestry Disability Questionnaire

${ }^{*} p$ is for the average scores over the observation period

exercise in combination with massage as a treatment option [18, 19]. The MDT, which prescribes repeated exercise in a specific direction along with an educational approach, is one of the strategies recommended by LBP guidelines [20].

A recent review and meta-analysis found moderate- to high-quality evidence that the MDT is superior to other rehabilitation interventions at reducing pain and disability in patients with chronic LBP; however, this depends on the type of intervention being compared with the MDT [21]. Moreover, a recent study showed that patients with chronic LBP treated using the MDT experienced only small improvements in pain intensity at shortterm follow-up compared to the effect of a placebo [22]. These results confirm the difficulty of treating a complex condition such as LBP with a single therapy, and strengthen the proposal-as already underlined in the guidelines for the treatment of LBP-to adopt integrative care plans instead of monodisciplinary care alone.

In line with this proposal, in the present study, decontracting massage was performed soon after MDT therapy, and systemic treatment with umPEA was added to these physical CAM therapies. umPEA treatment was maintained for 40 days after the physical CAM therapies were stopped. Massage has been the focus of several clinical trials, and an "evidence map" to depict the distribution of evidence available for massage visually as well as various pain indications for massage has recently been published. Therapeutic massage requires no special equipment; it can be administered almost anywhere, and there is a low likelihood of any serious harm from massage [23]. It is beneficial to patients with chronic LBP in terms of improving their symptoms and function. Decontracting massage invigorates and relaxes the body muscles through a deep pressure technique and energizing manual action. It is recommended as a preparation for any sport activity or to relieve muscle fatigue immediately after any sport activity.

The umPEA treatment played important roles in both the resolution of the pain and the consequent improvement in quality of life reported in the present study. PEA is a multiple anti-inflammatory lipid mediator that has been reported to have pain-relieving effects in various chronic pain conditions with different etiologies $[11,24]$. It is reported that PEA may exert both receptor-mediated and non-receptormediated effects at different cellular and tissue sites [25]. The transcription factor peroxisome proliferator-activated receptor alpha (PPAR- $\alpha)$ was identified as a possible target for the antiinflammatory action of PEA [26]. PEA may also interact with other members of the PPAR family to elicit its anti-inflammatory activity [27, 28]. PPARs are found on dorsal root ganglion sensory neurons and glial cells. PEA may activate these receptors and modulate both the perception and transmission of peripheral pain signaling and spinal pain amplification mechanisms, thereby exerting its influence on different types and phases of pain [29]. PEA potentiates the action of anandamide at cannabinoid 
(CB) receptors in a $\mathrm{CB} 2$ receptor antagonistsensitive fashion (while having no appreciable affinity for either CB1 or CB2 receptors) and the anandamide-induced desensitization of transient receptor potential cation channel subfamily $\mathrm{V}$ member 1 (TRPV1) channels ("entourage" effects). The direct activation and desensitization of TRPV1 channels are important mechanisms in the modulation of pain signaling [30, 31].

The principal limitation of this study is the lack of a comparison between the three treatment strategies. However, the group of patients examined in the present study reported that they had been in pain for about 2 years, even though they were using classic pain relief therapies. The complexity of the condition suffered by the patients necessitated the use of a combination of therapies.

\section{CONCLUSIONS}

The results of the present study suggest that PEA, especially when combined with other pharmacological or nonpharmacological therapies, could meet the need for a multitarget approach to tackling the as-yet unsolved problem of chronic pain resistant to conventional therapies [32].

\section{ACKNOWLEDGMENTS}

The authors thank Dr. Carlo Schievano for the statistical analysis, as well as the participants in the study.

Funding. No funding or sponsorship was received for this study or the publication of this article.

Authorship. All named authors meet the International Committee of Medical Journal Editors (ICMJE) criteria for authorship for this article, take responsibility for the integrity of the work as a whole, and have given their approval for this version to be published.
Authorship Contributions. Chiara Asaro drafted the manuscript. All authors have read and approved the final manuscript.

Disclosures. Giustino Varrassi is the Editorin-Chief of this journal. Dalila Scaturro, Chiara Asaro, Lorenza Lauricella, Sofia Tomasello, and Giulia Letizia Mauro have nothing to disclose.

Compliance with Ethics Guidelines. This study was performed in agreement with the Good Clinical Practice guidelines and the principles of the Declaration of Helsinki of 1964 and its subsequent revisions. The study was approved by the Polyclinic University Hospital Paolo Giaccone Ethics Committee (report number 05/2019). Written consent for participation in the study was obtained from each patient after they had been fully informed of the purpose of the study.

Data Availability. The data used to support the findings of this study are included within the article.

Open Access. This article is distributed under the terms of the Creative Commons Attribution-NonCommercial 4.0 International License (http://creativecommons.org/licenses/ by-nc/4.0/), which permits any noncommercial use, distribution, and reproduction in any medium, provided you give appropriate credit to the original author(s) and the source, provide a link to the Creative Commons license, and indicate if changes were made.

\section{REFERENCES}

1. Carlson BB, Albert TJ. Lumbar disc herniation: what has the Spine Patient Outcomes Research Trial taught us? Int Orthop. 2019;43(4):853-9. https:// doi.org/10.1007/s00264-019-04309-x.

2. Cunha C, Silva AJ, Pereira P, Vaz R, Gonçalves RM, Barbosa MA. The inflammatory response in the regression of lumbar disc herniation. Arthritis Res Ther. 2018;20(1):251. https://doi.org/10.1186/ s13075-018-1743-4.

3. Petit A, Roquelaure Y. Low back pain, intervertebral disc and occupational diseases. Int J Occup Saf 
Ergon. 2015;21(1):15-9. https://doi.org/10.1080/ 10803548.2015.1017940.

4. Checchia GA, Letizia Mauro G, Morico G, Oriente A, Lisi G, Polimeni V, Lucia M, Rainieri M. Observational multicentric study on chronic sciatic pain: clinical data from 44 Italian centers. Eur Rev Med Pharmacol Sci. 2017;21(7):1653-64.

5. Zhang B, Xu H, Wang J, Liu B, Sun G. A narrative review of non-operative treatment, especially traditional Chinese medicine therapy, for lumbar intervertebral disc herniation. Biosci Trends. 2017;11(4): 406-17. https://doi.org/10.5582/bst.2017.01199.

6. Peul WC, van den Hout WB, Brand R, Thomeer RT, Koes BW, Leiden-The Hague Spine Intervention Prognostic Study Group. Prolonged conservative care versus early surgery in patients with sciatica caused by lumbar disc herniation: two year results of a randomised controlled trial. BMJ. 2008;336(7657):1355-8.

7. Baber Z, Erdek MA. Failed back surgery syndrome: current perspectives. J Pain Res. 2016;9:979-87.

8. Kanodia AK, Legedza AT, Davis RB, Eisenberg DM, Phillips RS. Perceived benefit of complementary and alternative medicine (CAM) for back pain: a national survey. J Am Board Fam Med. 2010;23(3): 354-62.

9. Esposito E, Cuzzocrea S. Palmitoylethanolamide is a new possible pharmacological treatment for the inflammation associated with trauma. Mini Rev Med Chem. 2013;13(2):237-55.

10. Skaper SD, Facci L, Fusco M, Della Valle MF, Zusso $\mathrm{M}$, Costa B, et al. Palmitoylethanolamide, a naturally occurring disease-modifying agent in neuropathic pain. Inflammopharmacology. 2014;22(2): 79-94. https://doi.org/10.1007/s10787-013-0191-7.

11. Paladini A, Fusco M, Cenacchi T, Schievano C, Piroli A, Varrassi G. Palmitoylethanolamide, a special food for medical purposes, in the treatment of chronic pain: a pooled data meta-analysis. Pain Physician. 2016;19(2):11-24.

12. Petrosino S, Cordaro M, Verde R, Schiano Moriello A, Marcolongo G, Schievano C, et al. Oral ultramicronized palmitoylethanolamide: plasma and tissue levels and spinal anti-hyperalgesic effect. Front Pharmacol. 2018;9:249.

13. McKenzie RA. The lumbar spine. Mechanical diagnosis and therapy. Wellington: Spinal Publications NZ Ltd; 1981.

14. McKenzie RA. The cervical and thoracic spine. Mechanical diagnosis and therapy. Waikanae: Spinal Publications NZ Ltd; 1990.
15. Apolone G, Mosconi P. The Italian SF-36 Health Survey: translation, validation and norming. J Clin Epidemiol. 1998;51(11):1025-36.

16. Monticone M, Baiardi P, Vanti C, Ferrari S, Pillastrini P, Mugnai R, et al. Responsiveness of the Oswestry Disability Index and the Roland Morris Disability Questionnaire in Italian subjects with sub-acute and chronic low back pain. Eur Spine J. 2012;21(1):122-9.

17. Ghildayal N, Johnson PJ, Evans RL, Kreitzer MJ. Complementary and alternative medicine use in the US adult low back pain population. Glob Adv Health Med. 2016;5(1):69-78.

18. Wong JJ, Côté P, Sutton DA, Randhawa K, Yu H, Varatharajan S, et al. Clinical practice guidelines for the noninvasive management of low back pain: a systematic review by the Ontario Protocol for Traffic Injury Management (OPTIMa) Collaboration. Eur J Pain. 2017;21(2):201-16.

19. Shipton EA. Physical therapy approaches in the treatment of low back pain. Pain Ther. 2018;7(2): 127-37. https://doi.org/10.1007/s40122-018-0105$\mathrm{x}$.

20. Chetty L. A critical review of low back pain guidelines. Workplace Health Saf. 2017;65(9):388-94. https://doi.org/10.1177/2165079917702384.

21. Lam OT, Strenger DM, Chan-Fee M, Pham PT, Preuss RA, Robbins SM. Effectiveness of the McKenzie method of mechanical diagnosis and therapy for treating low back pain: literature review with metaanalysis. J Orthop Sports Phys Ther. 2018;48(6): 476-90.

22. Garcia AN, Costa LDCM, Hancock MJ, Souza FS, Gomes GVFO, Almeida MO, et al. McKenzie Method of Mechanical Diagnosis and Therapy was slightly more effective than placebo for pain, but not for disability, in patients with chronic nonspecific low back pain: a randomised placebo controlled trial with short and longer term follow-up. Br J Sports Med. 2018;52(9):594-600.

23. Miake-Lye IM, Mak S, Lee J, Luger T, Taylor SL, Shanman R, et al. Massage for pain: an evidence map. J Altern Complement Med. 2019. https://doi. org/10.1089/acm.2018.0282.

24. Gatti A, Lazzari M, Gianfelice V, Di Paolo A, Sabato E, Sabato AF. Palmitoylethanolamide in the treatment of chronic pain caused by different etiopathogenesis. Pain Med. 2012;13(9):1121-30.

25. Petrosino S, Di Marzo V. The pharmacology of palmitoylethanolamide and first data on the therapeutic efficacy of some of its new formulations. $\mathrm{Br}$ J Pharmacol. 2017;174(11):1349-65. 
26. Lo Verme J, Fu J, Astarita G, La Rana G, Russo R, Calignano A, et al. The nuclear receptor peroxisome proliferator-activated receptor-alpha mediates the anti-inflammatory actions of palmitoylethanolamide. Mol Pharmacol. 2005;67(1): $15-9$.

27. Costa B, Comelli F, Bettoni I, Colleoni M, Giagnoni G. The endogenous fatty acid amide, palmitoylethanolamide, has anti-allodynic and anti-hyperalgesic effects in a murine model of neuropathic pain: involvement of $\mathrm{CB}(1)$, TRPV1 and PPARgamma receptors and neurotrophic factors. Pain. 2008;139(3):541-50.

28. Paterniti I, Impellizzeri D, Crupi R, Morabito R, Campolo M, Esposito E, et al. Molecular evidence for the involvement of PPAR- $\delta$ and PPAR- $\gamma$ in antiinflammatory and neuroprotective activities of palmitoylethanolamide after spinal cord trauma. J Neuroinflammation. 2013;10:20. https://doi.org/ 10.1186/1742-2094-10-20.
29. Khasabova IA, Xiong Y, Coicou LG, Piomelli D, Seybold V. Peroxisome proliferator-activated receptor $\alpha$ mediates acute effects of palmitoylethanolamide on sensory neurons. J Neurosci. 2012;32(37):12735-43.

30. Ambrosino P, Soldovieri MV, Russo C, Taglialatela M. Activation and desensitization of TRPV1 channels in sensory neurons by the PPAR $\alpha$ agonist palmitoylethanolamide. $\mathrm{Br} \mathrm{J}$ Pharmacol. 2013;168(6): 1430-44.

31. De Petrocellis L, Davis JB, Di Marzo V. Palmitoylethanolamide enhances anandamide stimulation of human vanilloid VR1 receptors. FEBS Lett. 2001;506(3):253-6.

32. Maione S, Costa B, Di Marzo V. Endocannabinoids: a unique opportunity to develop multitarget analgesics. Pain. 2013;154(Suppl 1):S87-93. 